\title{
ARCS AND GEODESICS IN METRIC SPACES
}

BY

S. B. MYERS

1. In a general metric space, a geodesic arc is defined to be a shortest rectifiable arc joining its end points. In a compact metric space, or more generally in a metric space in which bounded sets are compact, Menger $\left.{ }^{(}\right)$ has shown that any two points joinable by a rectifiable arc are joinable by a geodesic arc. The method used is based on two principles. First, the equicontinuity of a family of parametrized curves of bounded length guarantees the existence of a limiting curve in the sense of Frechet. Second, arc length in a metric space is a lower semicontinuous functional of arcs.

In this paper is proved the existence of a limiting curve for a family of curves of bounded length in a locally compact, almost complete metric space, (provided that the initial points of the curves form a set compact in the space). From this it follows that in such a space every pair of points joinable by a rectifiable arc is joinable by a geodesic arc. Then applications are made to the class of geodesic metric spaces; that is, rectifiably arcwise connected metric spaces in which distance is identical with the greatest lower bound of arc length. This class contains all convex, complete metric spaces. The class of locally compact geodesic metric spaces contains all symmetric Finsler manifolds, in particular, all Riemannian manifolds. A series of results obtained by Hopf and Rinow $\left({ }^{2}\right)$ for Riemannian manifolds are here generalized to locally compact geodesic metric spaces; for example, in a locally compact geodesic metric space completeness is equivalent to the Weierstrass-Bolzano Theorem.

2. Let us recall some of the notions attached to the concept of a metric space $\left(^{3}\right)$. A set of elements (points) is called a metric space $M$ if a distance function $\rho$ of pairs of points is given satisfying the conditions (1) $\rho(A, B)>0$ for $A \neq B,(2) \rho(A, B)=0$ for $A=B$, (3) $\rho(A, B)=\rho(B, A)\left({ }^{4}\right)$, (4) $\rho(A, B)$ $+\rho(B, C) \geqq \rho(A, C)$. The space $M$ is provided with a Hausdorff topology by the open sphere neighborhoods of points of $M$. The distance function $\rho$ is uniformly continuous in this topology. $M$ is compact if every infinite set of points has a limit point in this topology, locally compact if every point $A$ has

Presented to the Society, August 14, 1944; received by the editors June 16, 1944.

(1) K. Menger, Math. Ann. vol. 103 (1930) p. 492.

(2) H. Hopf and W. Rinow, Comment. Math. Helv. vol. 3 (1931).

(3) For an excellent exposition of the modern development of abstract metrics, see L. M. Blumenthal, Distance geometries, University of Missouri Studies, April, 1938.

(4) The results of this paper hold with obvious modifications for non-symmetric metric spaces, which are spaces satisfying conditions (1), (2), (4), but not necessarily (3). 
a closed sphere neighborhood $\rho(A, P) \leqq \epsilon$ which is compact, complete if every Cauchy sequence converges. A set of points $E$ in $M$ is said to be bounded if for some point $P$ of $M$ (hence for every point of $M$ ) the distances from $P$ to the points of $E$ are uniformly bounded. $M$ is said to satisfy the WeierstrassBolzano (W.B.) Theorem if every infinite bounded set of points has a limit point in $M$.

A point $C$ is said to be between $A$ and $B$ if $\rho(A, C)+\rho(C, B)=\rho(A, B)$. If for every pair $A, B$ there is at least one point between $A$ and $B, M$ is called convex.

A subset $C$ of $M$ which is the image, under a continuous mapping $\mu$, of the straight line interval $I: 0 \leqq t \leqq 1$ is called a continuous curve (Peano space) with parametrization $\mu$. If $\mu$ is a homeomorphism, $C$ is called an arc. As is well known, every continuous curve is a locally connected compact continuum, and conversely. The length of a continuous curve for a parametrization $\mu$ is defined as follows. Consider a finite set $E$ of values of $t, t=0<t_{1}$ $<t_{2}<\cdots<t_{n}=1$, and let $P_{i}$ be the point in $C$ corresponding to $t_{i}$ under $\mu$. The l.u.b. $E<I \sum_{i=0}^{n-1} \rho\left(P_{i}, P_{i+1}\right)$, if finite, is called the $\mu$-length of $C$, and will be denoted by $l_{\mu}(C)$. $C$ is then said to be $\mu$-rectifiable. If $C$ is $\mu$-rectifiable, any subinterval $\bar{I}$ of $I$ defines a continuous subcurve $\gamma$ in $C$ which will also be $\mu$-rectifiable, and the $\mu$-length of $\gamma$ varies continuously with the end points of $\bar{I}$. The $\mu$-length of a continuous curve $C$ may vary with $\mu$. If $C$ is an arc, its length is unique for all parametrizations $\mu$ which are homeomorphsims. Furthermore, it is known that if $\alpha$ is any arc $A B$ in a continuous curve $C$, $l(\alpha) \leqq l_{\mu}(C)$ for all parametrizations $\mu$ of $C$. Also, $l(\alpha) \geqq \rho(A, B)$.

A well known property of length of continuous curves is lower semi-continuity. This means that if $P_{i}(t), 0 \leqq t \leqq 1$, is a sequence of rectifiably parametrized continuous curves such that $P_{i}(t)$ converges for each $t$ to a continuous curve $P(t)$, then $l[P(t)] \leqq \lim \inf l\left[P_{i}(t)\right]$ (we omit the subscript from the length notation whenever the parametrization is obvious).

A continuous curve $\alpha$ joining $A$ to $B$ is called a segment if $l(\alpha)=\rho(A, B)$. Every segment is an arc. It is known that a continuous curve is a segment if and only if for every triple of points $P_{1}, P_{2}, P_{3}$ ordered in the direction of increasing parameter, $P_{2}$ is between $P_{1}$ and $P_{3}$. Every subarc of a segment is a segment. A theorem of Menger guarantees that on a complete, convex metric space each pair of points is joinably by a segment.

An arc $\alpha$ with end points $A, B$ is called a geodesic arc if $l(\alpha)$ is at least as small as the length of any other arc joining $A$ and $B$; in other words, if $l(\alpha)=$ g.l.b. of lengths of arcs joining $A$ and $B$. We shall call the g.l.b. of lengths of arcs joining $A$ and $B$ the geodesic distance $\bar{\rho}(A, B)$. It is clear that $\rho(A, B) \leqq \bar{\rho}(A, B)$, so that a segment is always a geodesic arc, but not conversely.

3. For the sake of completeness we prove the following lemma, which is more or less well known. 
Lemma. In a compact metric space $M$ let $S$ be a set of continuous curves with the following properties:

(1) Each curve of $S$ can be parametrized rectifiably.

(2) The lower limit (lim inf) $l$ of lengths of curves of $S$ is finite.

Then a sequence of curves of $S$ can be selected, $C_{k}: P_{k}(u), 0 \leqq u \leqq 1$, such that $P_{k}(u)$ converges uniformly to a continuous curve $C: P(u)$, whose length is not greater than $l$.

Select a sequence $C_{i}: p_{i}(t)$ of curves of $S$ whose lengths $l\left(C_{i}\right)$ converge to $l$. If $l\left(C_{i}\right)=0$ for an infinite number of values of $i$, then the theorem is trivial; so we assume $l\left(C_{i}\right) \neq 0$. Reparametrize $C_{i}$ as follows: $u=l_{i}(0, t) / l\left(C_{i}\right)$ where $l_{i}(0, t)$ is the length of $C_{i}$ from $t=0$ to $t=t$. The curves $C_{i}$ now have the form $P_{i}(u), 0 \leqq u \leqq 1$.

Pick a denumerable everywhere dense set $u_{\alpha}$ of values of $u$ between 0 and 1 . The compactness of the space allows us to choose a subsequence $C_{j}$ of $C_{i}$ such that $P_{j}\left(u_{\alpha}\right)$ converges for each $\alpha$. Denote $\lim P_{j}\left(u_{\alpha}\right)$ by $P\left(u_{\alpha}\right)$.

Now the family of functions $P_{j}(u)$ is equicontinuous. For there is a longest curve $c$ of $C_{j}$, and if we denote by $l_{j}\left(\bar{u}, u^{*}\right)$ the length of $C_{j}$ from $u=\bar{u}$ to $u=u^{*}$,

But

$$
l_{j}\left(\bar{u}, u^{*}\right)=\left(u^{*}-\bar{u}\right) \cdot l\left(C_{j}\right) \leqq\left(u^{*}-\bar{u}\right) \cdot l(c) .
$$

$$
\rho\left[P_{j}(\bar{u}), P_{j}\left(u^{*}\right)\right] \leqq l_{j}\left(\bar{u}, u^{*}\right),
$$

hence if $u^{*}-\bar{u}<\epsilon / l(c)$, we see that

$$
\rho\left[P_{j}(\bar{u}), P_{j}\left(u^{*}\right)\right]<\epsilon \text { for all } j .
$$

From this equicontinuity we deduce the convergence of $P_{j}(u)$ for all $u$ as follows. Choose an arbitrary value of $u$, and let $u^{\prime}$ be one of the $u_{\alpha}$ 's such that

$$
\rho\left[P_{j}\left(u^{\prime}\right), P_{j}(u)\right]<\epsilon / 4 \text { for all } j .
$$

Now for all $j>N, \rho\left[P_{j}\left(u^{\prime}\right), P\left(u^{\prime}\right)\right]<\epsilon / 4$. Hence for all $j, k>N$,

$$
\rho\left[P_{j}(u), P\left(u^{\prime}\right)\right]<\epsilon / 2, \quad \rho\left[P_{k}(u), P\left(u^{\prime}\right)\right]<\epsilon / 2 .
$$

Thus $\rho\left[P_{j}(u), P_{k}(u)\right]<\epsilon$ for all $j, k>N$, so that $P_{j}(u)$ is a Cauchy sequence, hence convergent.

The uniform convergence of $P_{j}(u)$ and the continuity of the limit function $P(u)$ follow in standard fashion from the convergence and equicontinuity of $P_{j}(u)$. The lower semicontinuity of arc length guarantees that $l[P(u)] \leqq l$, and the lemma is proved.

We now introduce the notion of an almost complete metric space. Consider a sequence of points $P_{i}$ with the property that for an arbitrary $\epsilon>0$ there exists an $N$ such that for $i, j>N, P_{i}$ can be joined to $P_{j}$ by an arc of length $<\epsilon$. Such a sequence is clearly a Cauchy sequence, and will be called a strong Cauchy sequence. If every strong Cauchy sequence in $M$ converges, $M$ will be called almost complete. Completeness obviously implies almost completeness. 
As an example of an almost complete, but not complete, metric space consider the curve $M, y=x \sin (1 / x), x \neq 0$, in the $(x, y)$-plane, with the euclidean metric. The set of points $\left(1 / \pi^{n}, 0\right)$ is a non-convergent Cauchy sequence, hence $M$ is not complete. But clearly $M$ is almost complete.

THEOREM 3.1. In a locally compact, almost complete metric space $M$ let $S$ be a set of continuous curves with the following properties:

(1) Each curve of $S$ can be rectifiably parametrized.

(2) The lower limit $l$ of lengths of curves of $S$ is finite.

(3) The initial points of the curves of $S$ form a set of points compact in $M$. Then a sequence of curves of $S$ can be selected, $C_{k}: P_{k}(u), 0 \leqq u \leqq 1$, such that $l\left(C_{k}\right) \rightarrow l$ and $P_{k}(u)$ converges uniformly to a continuous curve $C: P(u)$ with length not greater than $l$.

Select a subsequence whose lengths converge to $l$, and then a subsequence $C_{i}$ whose initial points $A_{i}$ converge, say to $A$. Reparametrize $C_{i}$ by the parameter $u$ used in the proof of the lemma, so that $u=l_{i}(0, t) / l\left(C_{i}\right)$, and $C_{i}$ has the form $P_{i}(u)$.

Let us use the notation $r_{P}$ for the l.u.b. of the radii of compact sphere neighborhoods of the point $P$. Consider $N\left(A, 3 r_{A} / 4\right)$, the set of points $Q$ for which $\mathrm{P}(A, Q) \leqq 3 r_{A} / 4$. Let $u_{1}=\min \left[1, r_{A} / 2 l\right]$. Then for large $i$ the portion of $C_{i}$ defined by $0 \leqq u \leqq u_{1}$ is in $N\left(A, 3 r_{A} / 4\right)$. This is seen as follows. Let $l_{i}(0, u)$ be the length of $C_{i}$ from $u=0$ to $u=u$. Then for $u \leqq u_{1}$ and for large $i$

$$
\rho\left[A_{i}, P_{i}(u)\right] \leqq l_{i}(0, u) \leqq l_{i}\left(0, u_{1}\right)=u_{1} l\left(C_{i}\right) \leqq u_{1} \cdot l+\epsilon \leqq r_{A} / 2+\epsilon .
$$

Now

$$
\rho\left[A, P_{i}(u)\right] \leqq \rho\left(A, A_{i}\right)+\rho\left[A_{i}, P_{i}(u)\right] \leqq \epsilon+\rho\left[A_{i}, P_{i}(u)\right] \leqq r_{A} / 2+2 \epsilon
$$

for large $i$. Thus for $u \leqq u_{1}, P_{i}(u)$ lies in the compact space $N\left(A, 3 r_{A} / 4\right)$.

The curves $P_{i}(u)\left(0 \leqq u \leqq u_{1}\right)$ now satisfy the hypotheses of the lemma. Therefore we can pick a subsequence $P_{j}(u)$ converging to a continuous curve $P(u), 0 \leqq u \leqq u_{1}$. If $u_{1}=1$, the theorem is proved.

If $u_{1}<1$, denote $P_{j}\left(u_{1}\right)$ by $A_{j}^{1}, P\left(u_{1}\right)$ by $A^{1}$. Using $A^{1}$ instead of $A$ as the base point, repeat the process. We obtain a limiting continuous curve $P(u)$, $u_{1} \leqq u \leqq u_{2}$, where $u_{2}=\min \left[1, u_{1}+r_{A^{1}} / 2 l\right]$, and $P_{k}(u)$, a subsequence of $P_{j}(u)$, converges uniformly to $\dot{P}(u), u_{1} \leqq u \leqq u_{2}$. Combining the two steps of the process, $P_{k}(u)$ converges uniformly to $P(u)$ for $0 \leqq u \leqq u_{2}$.

Continuing in this manner, we extend the definition of $P(u)$ step by step, so that after $\alpha$ steps $P(u)$ is defined for $0 \leqq u \leqq u_{\alpha}$. It is clear that there cannot be a last value of $u<1$ for which $P(u)$ will be defined after a finite number of steps. We now show that there is no first value of $u$ for which $P(u)$ will not be defined.

Suppose there is such a first value $\bar{u}$. Then $u_{\alpha} \rightarrow \bar{u}$. Let $A_{k}^{(\alpha)}=P_{k}\left(u_{\alpha}\right)$, and $A^{(\alpha)}=P\left(u_{\alpha}\right)$. By the lower semicontinuity of arc length, the length of $P(u)$ 
from $u_{\alpha}$ to $u_{\beta}$ will be less than or equal to $\left|u_{\beta}-u_{\alpha}\right| \cdot l$. But $\left|u_{\beta}-u_{\alpha}\right|<\epsilon / l$ for $\alpha, \beta$ large, since $u_{\alpha} \rightarrow \bar{u}$. Thus $A^{(\alpha)}$ is a strong Cauchy sequence, and by the almost completeness assumption must converge, say to $\bar{A}$.

Let $\alpha$ be so large that $u_{\alpha+1}-u_{\alpha}<r_{\bar{A}} / 4 l$, and $\rho\left[A^{(\alpha)}, \bar{A}\right]<r_{\bar{A}} / 2$. Then $N\left[A^{(\alpha)}, r_{\bar{A}} / 2\right]<N\left(\bar{A}, r_{\bar{A}}\right)$, and so is compact. But since

$$
r_{\bar{A}} / 4 l>u_{\alpha+1}-u_{\alpha}=r_{A}^{(\alpha)} / 2 l,
$$

$r_{\bar{A}} / 2>r_{A^{(\alpha)}}$, which contradicts the fact that $N\left[A^{(\alpha)}, r_{\bar{A}} / 2\right]$ is compact.

Hence the definition of $P(u)$ is extendable by a finite number of steps to include $0 \leqq u \leqq 1$, and $C_{k}: P_{k}(u)$ converges uniformly to $C: P(u)$. Because of the lower semicontinuity of arc length, $l(C) \leqq l$, and the theorem is proved.

THEOREM 3.2. If in Theorem 3.1 the hypothesis of almost completeness is not made, then either (or both):

(1) The conclusion of Theorem 3.1 holds.

(2) $A$ sequence of curves of $S$ can be selected, $C_{k}: P_{k}(u), 0 \leqq u \leqq 1$, with lengths converging to $l$, such that for some $\bar{u} \leqq 1, P_{k}(u)$ converges uniformly for $0 \leqq u<\bar{u}$ to a limiting half-open continuous curve (that is, continuous image of the interval $0 \leqq u<\bar{u}) C: P(u)$, which is closed in $M$ and of length not greater than $\bar{u} \cdot l$.

To prove this, use the process described in the proof of Theorem 3.1. This process will fail to reach $u=1$ if and only if the points $A^{(\alpha)}$ form a non-convergent strong Cauchy sequence. In that case, a denumerable number of steps are involved in the process, and a denumerable number of choices of subsequences must be made to arrive at the subsequence $P_{k}(u)$. In spite of the openness of the interval $0 \leqq u<\bar{u}$, the uniform convergence of $P_{k}(u)$ and the continuity of $P(u)$ for $0 \leqq u<\bar{u}$ follow from the equicontinuity of the family $P_{k}(u)$.

THEOREM 3.3. If in Theorem 3.1, the hypothesis (2) is replaced by the assumption that the lengths of the curves of $S$ have $\lim$ inf $=\infty$, then a sequence of curves of $S$ can be selected, $C_{k}: P_{k}(s), 0 \leqq s \leqq l\left(C_{k}\right)$, such that $P_{k}(s)$ converges to a half-open continuous curve $C: P(s), 0 \leqq s<\infty$, every closed subcurve of which is rectifiable, and this convergence is uniform over any closed subinterval $0 \leqq s \leqq \bar{s}$.

Select a sequence $C_{i}$ whose initial points converge to a point $A$. Using length $s$ as parameter along each $C_{i}$, for each $s, P_{i}(s)$ is defined for almost all $i$. The process of Theorem 3.1 is now modified so that in the first step we obtain the uniform convergence of a subsequence $C_{j}$ to a continuous curve $C: P(s)$ for $0 \leqq s \leqq \bar{s}$, where $\bar{s}=r_{A} / 2$. Continuing the process as before, there is clearly no last value of $s$ reachable by this process, and by the same reasoning as in Theorem 3.1 almost completeness guarantees that there is no first finite value of $s$ not reachable by a finite number of steps. Hence after a denumerable number of steps we obtain a subsequence of the desired sort. 
THEOREM 3.4. If, in Theorem 3.3, the hypothesis of almost completeness is not made, then either the conclusion of Theorem 3.3 holds, or else a sequence of curves of $S$ can be selected, $C_{k}: P_{k}(s), 0 \leqq s \leqq l\left(C_{k}\right)$, such that for $0 \leqq s<\bar{s}(\bar{s}<\infty)$, $P_{k}(s)$ converges uniformly to a half-open continuous curve $C: P(s), 0 \leqq s<\bar{s}$, which is closed in $M$ and whose length is not greater than $\bar{s}$.

The proof is left to the reader.

4. A first application of Theorem 3.1 is to the set of rectifiable arcs joining a pair of points.

THEOREM 4.1. In a locally compact, almost complete metric space $M$, any two points which can be joined by a rectifiable arc can be joined by a geodesic arc.

Consider $\bar{\rho}(A, B)$, the g.l.b. of lengths of rectifiable arcs joining $A$ and $B$. Assume this g.l.b. is a lim inf (otherwise the theorem is obvious). According to Theorem 3.1, a sequence of rectifiable arcs $A B$ can be selected, with lengths converging to $\bar{\rho}(A, B)$, converging uniformly to a continuous curve $C$ joining $A$ and $B$, of length not greater than $\bar{\rho}(A, B)$. $C$ must be an arc of length $\bar{\rho}(A, B)$, hence a geodesic; otherwise it would contain an arc $A B$ of length less than $\bar{\rho}(A, B)$, which is impossible. This proves the theorem.

If $M$ is not almost complete, the half-open continuous curve issuing from $A$ whose existence is guaranteed by Theorem 3.2 may be a half-open arc without being a geodesic. For example, consider the set of points $M$ in the $(x, y)$-plane consisting of the parabolic arcs $y+(1+1 / n)=(1+1 / n) x^{2}$, $n=1,2,3, \cdots,-1 \leqq x \leqq 1$, joining $(-1,0)$ to $(1,0)$, together with the points $(x, y)$ satisfying the inequalities

$$
-1 \leqq x<0, \quad x^{2}-1 \leqq y \leqq-1-x
$$

and the points satisfying the inequalities

$$
0<x \leqq 1, \quad x^{2}-1 \leqq y \leqq x-1 .
$$

This set of points, with euclidean distance as metric, forms a locally compact, but not almost complete, metric space. The limit arc $y+1=x^{2}(-1 \leqq x<0)$ is not a geodesic; in fact, no subarc of it is a geodesic, since each pair of its points is joinable by a straight line segment.

THEOREM 4.2. In a locally compact, almost complete, metric space $M$, let $E_{1}$ and $E_{2}$ be two compact sets. If $E_{1}$ and $E_{2}$ can be joined by a rectifiable arc, they can be joined by a shortest arc.

The proof is left to the reader.

A set of points $E$ will be said to be bounded with respect to geodesic distance if there exists a point $P$ such that the geodesic distance from $P$ to the points of $E$ exists and is bounded. This is equivalent to the condition that $P$ be joinable to the points of $E$ by rectifiable arcs of uniformly bounded length.

As is well known, a metric space may be locally compact and complete 
without satisfying the Weierstrass-Bolzano theorem. However, the following theorem shows that local compactness and completeness, in fact, local compactness and almost completeness, imply a weak form of the W.B. theorem.

THEOREM 4.3. In a locally compact, almost complete metric space $M$ every infinite set $E$ of points bounded with respect to geodesic distance has a limit point.

Consider a set $S$ of rectifiable arcs of bounded length joining $P$ to the points of $E$. By Theorem 3.1, a sequence $C_{i}$, converging to a limit curve, can be selected. The convergence of the final points of $C_{i}$ gives the desired result.

5. We have already defined the geodesic distance $\bar{\rho}(A, B)$ to be the g.l.b. of length of arcs joining $A$ and $B$. If we now assume that $M$ is rectifiably connected, that is, that every pair of points on $M$ is joinable by a rectifiable arc, then $\bar{\rho}$ is defined for every pair of points of $M$. It is easily shown that this distance function $\bar{\rho}$ satisfies the usual metric axioms, and thus defines a new metric space $\bar{M}$ on the points of $M$. Let us consider some of the relations between $M$ and $\bar{M}$, which we shall call the associated metric space of $M$.

Topologically, the two spaces may be quite different. $M$ may be compact without $\bar{M}$ being compact. However, due to the relation $\bar{\rho}(A, B) \geqq \rho(A, B)$, the identity mapping from $\bar{M}$ to $M$ is continuous. Thus, if $\bar{M}$ is compact, $M$ is compact. Local compactness of $\bar{M}$ does not imply and is not implied by local compactness of $M$, as we shall see by examples later. An arc in $M$ is not necessarily an arc in $\bar{M}$, and a connected subset of $M$ is not necessarily connected in $\bar{M}$. For example, consider the set of points in the $(x, y)$-plane consisting of the non-rectifiable arc

$$
y=x \sin (1 / x), \quad 0<x \leqq 1 / \pi, \quad y=0, \quad x=0
$$

and the circle $(x-1 / 2 \pi)^{2}+y^{2}=1 / 4 \pi^{2}$. This set, with euclidean distance, forms a metric space $M$ which is rectifiably arcwise connected. In $M$, the set

$$
y=x \sin (1 / x), \quad 0<x \leqq 1 / \pi, \quad y=0, \quad x=0
$$

is an arc, but in $\bar{M}$ it is not even connected.

$\bar{M}$ is always locally connected. In fact, every sphere neighborhood $N$ of every point $P$ of $\bar{M}$ has the property that every point inside $N$ can be joined to $P$ by an arc inside $N$. Thus a compact $\bar{M}$ is a Peano space.

A necessary and sufficient condition that $M$ and $\bar{M}$ be homeomorphic under the identity mapping is that for every point $P$ of $M$, corresponding to an arbitrary $\epsilon>0$, there exists a neighborhood $N(P)$ in $M$ such that every point in $N(P)$ can be joined to $P$ by an arc of length less than $\epsilon$.

Length in $\bar{M}$ is the same as in $M$; more precisely a rectifiable arc in $M$ is a rectifiable arc in $\bar{M}$ and conversely, and $l(\gamma)=\bar{l}(\gamma)$. Hence geodesic distances are the same in $M$ and $\bar{M}$, and $M$ and $\bar{M}$ have all geodesic arcs in common, all these goedesic arcs being segments in $\bar{M}$. It follows that the associated metric space $M^{*}$ of $\bar{M}$ is metrically identical with $\bar{M}$. In fact, we have: 
THEOREM 5.1. A necessary and sufficient condition that a rectifiably connected metric space $M$ be metrically identical with its associated metric space $\bar{M}$ is that $M$ be an associated metric space itself.

The sufficiency of the condition follows from the relation $M^{*}=\bar{M}$. The necessity follows from the fact that if $M=\bar{M}, M$ is its own associated space.

Thus the class of associated metric spaces is identical with the class of rectifiably connected metric spaces identical with their own associates. This is the class of metric spaces for which $\rho=\bar{\rho}$, and will be called the class of geodesic metric spaces. It includes all convex, complete metric spaces since on such a space each pair of points is joinable by a segment, hence $\rho=\bar{\rho}$.

In a geodesic metric space, the notions of completeness and almost completeness are identical. A surprising result is the following.

THEOREM 5.2. A rectifiably connected metric space $M$ is almost complete if and only if its associated metric space $\bar{M}$ is complete.

If $\bar{M}$ is complete, every Cauchy sequence converges in $\bar{M}$. Every strong Cauchy sequence in $M$ is a Cauchy sequence in $\bar{M}$; convergence in $\bar{M}$ implies convergence in $M$. Thus completeness of $\bar{M}$ implies almost completeness of $M$.

Now suppose $M$ is almost complete. Let $P_{i}$ be a Cauchy sequence in $\bar{M}$; since it is a strong Cauchy sequence in $M$, it converges in $M$, say to $P$. Let us show it also converges in $\bar{M}$. For an arbitrary $\epsilon>0$ there exists an $N_{\epsilon}$ such that $i, j>N_{\epsilon}$ implies the existence of an arc of length less than $\epsilon$ joining $P_{i}$ and $P_{j}$. Let $P_{i_{0}}$ be any member of $P_{i}$ such that $i_{0}>N_{\epsilon}$, and let $i_{\alpha}$ be a sequence of integers such that $i_{\alpha}>N_{\epsilon / 2}{ }^{\alpha}$. Let $\gamma_{\alpha}$ be an arc of length less than $\epsilon / 2^{\alpha}$ joining $P_{i_{\alpha}}$ to $P_{i_{\alpha+1}}$. Then $\left[\sum_{\alpha=0}^{\infty} \gamma_{\alpha}\right]+P$ contains a rectifiable arc of length $2 \epsilon$ joining $P_{i_{0}}$ to $P$. Thus $P_{i} \rightarrow P$ in $\bar{M}$, and $\bar{M}$ is complete.

Corollary. If $M$ is complete, $\bar{M}$ is complete $\left(^{5}\right)$.

If $\bar{M}$ is complete, $M$ is not necessarily complete. For example, the halfopen arc $y=x \sin (1 / x), 0<x \leqq 1 / \pi$, with euclidean distance, is an $M$ which is not complete, yet $\bar{M}$ is complete.

6. The theorems of $\$ 4$ may now be rephrased as follows.

THEOREM 6.1. If a rectifiably connected metric space $M$ is locally compact, and $\bar{M}$ is complete, then every pair of points in $M$ can be joined by a geodesic arc.

THEOREM 6.2. If a rectifiably connected metric space $M$ is locally compact, and $\bar{M}$ is complete, then any two sets compact in $M$ can be joined by a shortest arc.

THEOREM 6.3. If a rectifiably connected metric space $M$ is locally compact, and $\bar{M}$ is complete, then every infinite set of points bounded in $\bar{M}$ has a limit point in $M$.

(5) An erroneous counterexample to this theorem has been given. See footnote 3, p. 119. 
If we now apply Theorem 6.1 to the space $\bar{M}$, and recall that a geodesic arc in $\bar{M}$ is a geodesic arc in $M$ and that $M^{*}=\bar{M}$, we obtain the following theorem.

THEOREM 6.4. If $M$ is a rectifiably connected metric space and $\bar{M}$ is locally compact and complete, then every pair of points in $M$ can be joined by a geodesic arc.

It has now been shown that any of the following four hypotheses is sufficient to guarantee the joinability by a geodesic arc of any pair of points on a rectifiably connected metric space $M$.

(a) $M$ is locally compact and complete.

(b) $M$ is locally compact and $\bar{M}$ is complete.

(c) $\bar{M}$ is locally compact and $M$ is complete.

(d) $\bar{M}$ is locally compact and complete.

Of these four conditions, (a) $\rightarrow$ (b), and (c) $\rightarrow$ (d), but they are otherwise independent. An example of a compact $M$ whose $\bar{M}$ is not locally compact is the set of points in the $(x, y)$-plane consisting of the straight line segments joining the points $(1 / n, 0), n=1,2, \cdots$, to the point $(0,1)$ plus the $y$-axis $0 \leqq y \leqq 1$. In this example, $\bar{M}$ is not locally compact at $(0,1)$. This same example, with $(0,0)$ omitted, serves to illustrate a locally compact $M$ whose $\bar{M}$ is neither locally compact nor complete. An example in which $\bar{M}$ is locally compact and complete, and $M$ is neither locally compact nor complete, is the following. Consider the set of points in the $(x, y)$-plane consisting of the curve $y=x \sin (1 / x), 0<x \leqq 1 / \pi$, the segment $y=1,0 \leqq x \leqq 1 / \pi$, the segment $x=1 / \pi$, $0 \leqq y \leqq 1$, and the segments $x=1 / n \pi, n=2,3, \cdots, 0 \leqq y \leqq 1-1 / n$. The set $M$ with euclidean distance fails to be locally compact at $(0,1)$, and is clearly not complete; but $\bar{M}$ is both locally compact and complete.

When (a) or (b) is assumed, the geodesic arc which results is the uniform limit of a minimizing sequence only in the topology of $M$; whereas when (c) or (d) is assumed, the uniform convergence of the minimizing sequence is in the strong sense of geodesic distance.

7. We now confine our attention to geodesic metric spaces. The theorems of this section generalize to the class of locally compact geodesic metric spaces theorems proved by Hopf and Rinow, loc. cit., for the case of Riemannian manifolds $\left(^{6}\right)$.

THEOREM 7.1. In a locally compact, complete geodesic metric space each pair of points can be joined by a segment.

This follows from Theorem 4.1 and the fact that a geodesic arc in a geodesic metric space is a segment.

Theокем 7.2. In a geodesic metric space, local compactness plus completeness are equivalent to the Weierstrass-Bolzano theorem.

(8) The theorems of this section are related to results of S. Cohn-Vossen, Compositio Math. vol. 3 (1936) pp. 441-452. 
This follows from Theorem 6.3.

TheOREm 7.3. A locally compact geodesic metric space $M$ is convex.

Consider two points $A$ and $B$ of $M$. Let $C_{i}$ be a sequence of arcs joining $A$ and $B$ such that $l\left(C_{i}\right) \rightarrow \rho(A, B)$. Let $r$ be the radius of a compact neighborhood of $A$, and let $r / 2 \rho(A, B)=m$. Let $\gamma_{i}$ be the subarc of $C_{i}$ issuing from $A$ of length $m \cdot l\left(C_{i}\right)$, and let $P_{i}$ be the final end point of $\gamma_{i}$. Now $\rho\left(A, P_{i}\right) \leqq l\left(\gamma_{i}\right)=m \cdot l\left(C_{i}\right) \rightarrow r / 2$. Therefore almost all $P_{i}$ lie in $N(A, r)$. Hence $P_{i}$ has a limit point $P$, and a subsequence $P_{\alpha} \rightarrow P$, with $\rho(A, P) \leqq r / 2$.

Now let $g_{\alpha}$ be the subarc of $C_{\alpha}$ from $P_{\alpha}$ to $B$. Then

$$
\begin{aligned}
& \rho\left(P_{\alpha}, B\right) \leqq l\left(g_{\alpha}\right), \quad \rho\left[\left(P_{\alpha}, B\right) \rightarrow \rho(P, B)\right], \\
& l\left(g_{\alpha}\right)=(1-m) \cdot l\left(C_{\alpha}\right) \rightarrow \rho(A, B)-r / 2 .
\end{aligned}
$$

Therefore $\rho(P, B) \leqq \rho(A, B)-r / 2$. Combining this with $\rho(A, P) \leqq r / 2$, we obtain

$$
\rho(A, P)+\rho(P, B) \leqq \rho(A, B)
$$

so that $\rho(A, P)+\rho(P, B)=\rho(A, B)$ and $P$ is between $A$ and $B$. Thus $M$ is convex.

A convex geodesic metric space is not necessarily locally compact. For example, the segments in the $(x, y)$-plane joining $(0,1)$ to $(1 / n, 0)$ and to $(0,0)$, when metrized by the g.l.b. of arc length, form a convex but not locally compact $M$.

Theorem 7.1 could have been proved by using Theorem 7.3 and Menger's theorem that in a complete convex metric space every pair of points is joinable by a segment.

By a ray we shall mean a half-open arc, closed in $M$, every subarc of which is a segment. It is clear that a ray can exist only in a non-compact $M$, and a ray of finite length can exist only in an $M$ which is not almost complete.

TheOREM 7.4. In a locally compact geodesic metric space $M$, let $A$ and $B$ be any two points. Then either $A$ and $B$ can be joined by a segment, or else there exists a ray issuing from $A$ all of whose points are between $A$ and $B$ and whose length is less than $\rho(A, B)$. (Both possibilities may hold.)

If $A$ cannot be joined to $B$ by a segment, Theorem 3.2 guarantees the existence of a half-open continuous curve $C: P(u)$ issuing from $A$, closed in $M$, with the following properties:

(1) $P(u)$ is defined for $0 \leqq u<\bar{u}$, where $\bar{u}<1$.

(2) A sequence of arcs $C_{k}: P_{k}(u)$ joining $A$ and $B$ can be selected, whose lengths converge to $\rho(A, B)$ such that for $0 \leqq u<\bar{u}, P_{k}(u)$ converges uniformly to $C: P(u)$, and such that the length of $C_{k}$ from $A$ to $P_{k}(u)$ bears the ratio $u$ to the length of $C_{k}$.

It follows from (2) that 


$$
\rho[A, P(u)]=\lim \rho\left(A, P_{k}\right) \leqq \lim \left[u \cdot l\left(C_{k}\right)\right]=u \cdot \rho(A, B)
$$

and that

$$
\rho[P(u), B]=\lim \rho\left[P_{k}, B\right] \leqq \lim \left[(1-u) \cdot l\left(C_{k}\right)\right]=(1-u) \cdot \rho(A, B) .
$$

Hence

$$
\rho[A, P(u)]+\rho[P(u), B]=\rho(A, B)
$$

and $P(u)$ is between $A$ and $B$. Thus every point of $C$ is between $A$ and $B$.

Furthermore, it follows that $\rho[A, P(u)]=u \cdot \rho(A, B)$. But by lower semicontinuity the length of $C$ from $A$ to $P(u)$ is not greater than $u \cdot \rho(A, B)$, and so this length must equal $\rho[A, P(u)]$. Thus $C$ is a segment from $A$ to any of its points, hence $C$ is a ray.

THEOREM 7.5. A necessary and sufficient condition that a locally compact geodesic metric space $M$ be non-complete is that from every point $A$ issues a ray of finite length.

If $M$ contains a ray of finite length it is clearly non-complete.

If $M$ is not complete, let $P_{i}$ be a Cauchy sequence without limit point. Let $A$ be any point of the space. Either $A$ can be joined to each $P_{i}$ by a segment or else according to Theorem 7.4 a ray of finite length issues from $A$. If $A$ can be joined to each point $P_{i}$ by a segment $g_{i}$, since $P_{i}$ is a bounded sequence $l\left(g_{i}\right)$ is bounded, and the members of $g_{i}$ satisfy the hypothesis of Theorem 3.2. The first possible conclusion of Theorem 3.2 obviously does not hold, since $P_{i}$ has no limit point, hence the second conclusion does hold. The limiting curve $g: P(u)$ will be a ray, since for any of its subcurves $\left(u_{1}, u_{2}\right)$

$$
\begin{aligned}
l\left[P\left(u_{1}\right), P\left(u_{2}\right)\right] & \leqq \lim l\left[P_{k}\left(u_{1}\right), P_{k}\left(u_{2}\right)\right] \\
& =\lim \rho\left[P_{k}\left(u_{1}\right), P_{k}\left(u_{2}\right)\right]=\rho\left[P\left(u_{1}\right), P\left(u_{2}\right)\right] .
\end{aligned}
$$

THEOREM 7.6. A necessary and sufficient condition that a locally compact geodesic metric space $M$ be non-compact is that from every point $A$ issues a ray.

If $M$ contains a ray, it is clearly non-compact.

If $M$ is not compact and non-complete, by the previous theorem it contains a ray. If $M$ is non-compact but complete, let $P_{i}$ be a sequence without limit point. Let $A$ be any point of the space $M$, and $g_{i}$ segments joining $A$ to $P_{i}$, whose existence is affirmed by Theorem 7.1. By Theorem 7.2, $P_{i}$ contains no bounded sequence, hence $l\left(g_{i}\right) \rightarrow \infty$. Thus the segments $g_{i}$ form a family satisfying the hypotheses of Theorem 3.3. The limiting curve guaranteed by Theorem 3.3 will be a ray.

UNIVERSITY OF MICHIGAN, ANN ARBOR, Mich. 